\section{S15.2 SOCIAL NETWORKING AND DIFFUSION OF RISKS AND INTERVENTIONS AMONG YOUTH}

doi:10.1136/sextrans-2013-051184.0071

S D Young. University of California, Los Angeles, Los Angeles, CA, United States

Use of online social networking technologies has been growing rapidly, especially among groups at risk for sexually transmitted infections (STIs), such as men who have sex with men (MSM), homeless youth, and African American and Latino populations. International studies are beginning to suggest a number of important relationships between social networking technologies, at-risk populations, and risk for sexually transmitted infections: (1) At-risk populations are using social networking technologies to meet sexual partners, (2) Although at-risk populations who use social networking technologies are engaging in high rates of sexual intercourse, they are engaging in prevention behaviours that might mitigate their risk, and ( 3 ) Social networking technologies can be used as platforms for scaling and diffusing STI prevention and testing interventions. Online social networks are tools that can be used to rapidly spread information and social norms. These same technologies that can be used to potentially spread STI transmission must also be implemented as tools for preventing transmission. Recent research is discussed.

\section{S15.3 MODIFICATION OF SEXUAL NETWORKS AND SEXUAL RISK FOR MEN HAVING SEX WITH MEN (MSM) BY THE INTERNET}

doi:10.1136/sextrans-2013-051184.0072

U Marcus, ${ }^{2} \mathrm{~A} J$ Schmidt. 'Robert Koch-Institut, Berlin, Germany; ${ }^{2}$ Sigma Research, Department of Social \& Environmental Health Research, London School of Hygiene and Tropical Medicine, London, UK

The Internet provides new opportunities for sexual minorities to communicate and interact. Access to internet may facilitate coming-out for young MSM, sexual identity formation, and community building. On the other side the internet partly substitutes social and sexual venues where MSM congregate. Population and individual level aspects of internet communication among MSM were analysed based on data collected in the European MSM Internet Survey (EMIS).

EMIS was a large collaborative project of public health, academia and community based organisations from 35 European countries. The survey was online from June through August 2010, advertised on a large range of MSM websites. The questionnaire anonymously collected data from MSM across Europe. Among others, detailed information on the last sexual intercourse with a non-steady partner was collected.

The analysis is based on 174,209 eligible respondents. Participation rates in 38 countries with more than 100 respondents varied considerably (0.3 - 6.8/10,000 inhabitants) and correlated with internet access. A comparison of self-reported new diagnoses of HIV and national surveillance data suggests different relative sizes of MSM populations in different countries. Among men reporting a non-steady sex partner in the last 12 months, 58\% had met this partner on the internet. Compared with partners met in venues, serostatus communication with internet partners was more frequent, respondents more often already had sex with their internet partners before, and partners were more often presumed HIV seroconcordant. Contrastingly, refraining from anal sex was less often an option for partners met on the internet.

Improved access to internet may increase the relative size of MSM populations by involving a larger number of individuals into sexual networks. With broadening access to internet risk reducing aspects like increased communication before sexual encounters become more pronounced. Promoting protective and preventive behaviours can counter adverse effects of the internet on the HIV epidemic.

\section{S.16 - Challenging clinical conditions}

\section{S16.1 RECURRENT AND PERSISTENT URETHRITIS IN MEN - A STRUCTURED EVIDENCE BASED APPROACH TO MANAGEMENT}

doi:10.1136/sextrans-2013-051184.0073

\section{P Horner. University of Bristol, Bristol, UK}

This occurs in 10-20\% of men treated for acute NGU. Its aetiology is multifactorial, an infectious agent being identified in $<50 \%$ cases. Mycoplasma genitalium has been identified in $20-40 \%$ and Chlamydia trachomatis in up to $20 \%$. Ureaplasma urealyticum may also play a role. Trichomonas vaginalis is identified in up to $10 \%$ in populations where it is endemic.

Until recently azithromycin 1 grm and doxycycline $100 \mathrm{mgs}$ bd 7 days were considered equally effective in treating men with acute NGU. However this is not the case. The microbiological failure rate of azithromycin $1 \mathrm{grm}$ is $13-30 \%$ for $M$. genitalium and associated with $23 \mathrm{~S}$ rRNA gene macrolide antimicrobial resistance mutations, which it can induce. This has also been demonstrated to occur women. A prolonged course of azithromycin for 5 days appears to be effective but not always, possibly because of prior macrolide resistance. Up to $20 \%$ of men with Chlamydia will also fail azithromycin 1 grm but do not develop antimicrobial resistance mutations. Doxycycline $100 \mathrm{mgs}$ bd 7 days has a failure rate $>50 \%$ with $M$. genitalium but probably $<5 \%$ with Chlamydia. It does not induce antimicrobial resistance mutations. Ofloxacin is probably effective against Chlamydia but has a high failure rate $(\sim 50 \%)$ against $M$. genitalium and may result in quinolone antimicrobial resistance mutations. Moxifloxacin is effective against both micro-organisms.

Any treatment of recurrent/persistent NGU should cover $M$. genitalium and T. vaginalis. As there is a significant risk of macrolide and quinolone resistance developing in $M$. genitalium, an infection for which there is no commercial test, the most sensible strategy would be to use doxyccline $100 \mathrm{mgs}$ bd 7 dys as first line treatment and when using azithromycin to restrict prescribing to a five day course for both index cases and partners. The use of quinolones should be limited to moxifloxacin in those who fail azithromycin 5 dys treatment.

\section{S16.2 RECURRENT BV}

doi:10.1136/sextrans-2013-051184.0074

J Marrazzo. University of Washington, Seattle, WA, United States

Bacterial vaginosis (BV) is a common cause of vaginitis and increases women's risk of pelvic inflammatory disease, adverse pregnancy outcomes, and risk of STD/HIV acquisition. The aetiology of BV is unclear, though it is believed to involve loss of vaginal hydrogen peroxide-producing lactobacilli and acquisition of complex bacterial communities that include many fastidious BV-associated bacteria (BVAB) that have recently been detected using PCR methods. Treatment failure (persistence) is common, and may be facilitated by unprotected sex. Potential contributions to BV and BV persistence include (1) sexual partners as a reservoir for BVAB; (2) specific sexual practises, including male partners' condom use; and (3) the composition of the vaginal microbiota involved in BV. Specific BVAB in the Clostridiales Order may predict BV persistence when detected pretreatment, and have been detected in men whose female partners have BV. BVAB may be associated with unprotected sexual behaviour and failure of $\mathrm{BV}$ to resolve in women, supporting the hypothesis that BVAB colonisation of male genitalia may serve as a reservoir for re-infection of female partners. Moreover, specific sexual practises may favour vaginal colonisation with certain BVAB that have been associated with persistence. This session will provide background on $\mathrm{BV}$, and discuss the epidemiologic and microbiologic 
data to support a role for acquisition of BVAB and how this process might differ among subsets of women.

\section{S16.3 RECURRENT TV: THE POTENTIAL OF MOLECULAR TECHNIQUES TO IMPROVE CLINICAL PRACTISE}

doi:10.1136/sextrans-2013-051184.0075

M M Hobbs. University of North Carolina at Chapel Hill, Chapel Hill, NC, United States

Infection with the widespread protozoan pathogen Trichomonas vaginalis (TV) does not result in lasting immunity, and recurrent infections are common. Whether due to unrecognised, inadequately treated or repeatedly acquired TV infections, recurrent vaginitis in women, nongonococcal urethritis and chronic prostatitis in men are well-recognised and challenging clinical conditions. Despite increased recognition in the STI research community of the adverse consequences of trichomoniasis in women and men and the potential for TV infection to increase transmission of HIV and other STIs, T. vaginalis infection remains underappreciated by clinicians, public health professionals, policy makers and patients. Trichomoniasis is not a reportable STI in most countries, and TV infection is often asymptomatic, thus many infections are neither diagnosed nor treated. Symptomatic infection in women is the clinical presentation most likely to be recognised and treated, but treatment of male sexual partners of infected women is infrequent or inadequate, and testing and treatment of trichomoniasis in male patients is rare. Thus, reservoirs of infection persist.

Recent improvements in molecular diagnostics for detection of TV in women and men have the potential to improve clinical practise. Rapid antigen detection tests offer point of care testing and improved treatment options in settings where technically complex and costly nucleic acid amplification tests (NAATs) are not available, and NAATs offer highly sensitive and specific testing options for detection of TV in urogenital specimens commonly tested for other sexually transmitted pathogens including $N$. gonorrhoeae and C. trachomatis. With enhanced awareness, availability and application of these molecular tests, better detection and treatment of trichomoniasis in women and in their sexual partners can be achieved with eventual reduction of the adverse reproductive consequences associated with $T$. vaginalis infection.

\section{S16.4 LYMPHOGRANULOMA VENEREUM IN MEN WHO HAVE SEX WITH MEN. AN ONGOING EPIDEMIC SINCE 10 YEARS, BUT STILL NOT TACKLED}

doi:10.1136/sextrans-2013-051184.0076

\begin{abstract}
1,2,3H J C De Vries. 'STI outpatient clinic, Public Health Service Amsterdam, Amsterdam, The Netherlands; ' 2 Dermatology, Academic Medical Centre, University of Amsterdam, Amsterdam, The Netherlands; ${ }^{3}$ Centre for Infection and Immunity (CINIMA), Academic Medical Centre, University of Amsterdam, Amsterdam, The Netherlands
\end{abstract}

LGV is endemic in large parts of the tropics. Since 2003 anorectal LGV is also endemic among Men who have Sex with Men (MSM) throughout the industrialised world. Currently we see an increase in de incidence of LGV cases among MSM in Amsterdam. Occasional cases of heterosexual LGV are usually imported from endemic countries.

LGV is caused by Chlamydia trachomatis (Ct) biovar L. Compared to non-L biovar infections, LGV has a completely different clinical picture characterised by an invasive, lymph destructive and fibrosing inflammatory reaction. The majority of MSM with LGV are HIV co-infected (up to to $85 \%$ ), and a considerable portion is hepatitis $\mathrm{C}$ co-infected.

LGV requires extensive treatment in contrast to non-L Ct infections, thus correct biovar identification is clinically relevant. Routinely LGV is excluded in Ct positive anal, ulcer, and bubo samples. Urethral LGV is not screened routinely. The vast majority of reported LGV cases comprise anorectal infections. Infections residing at other locations than the rectum could form an undiagnosed and undertreated reservoir contributing to ongoing LGV transmission. We recently found concurrent urethral LGV infections in $2.1 \%$ of MSM with anorectal LGV. Moreover, $6.8 \%$ of the partners of anorectal LGV cases had a urethral LGV infection. This shows that urethral LGV is common, probably key in transmission, and missed in current routine LGV screening algorithms.

In European MSM the majority of LGV infections is caused by biovar L2b (Amsterdam variant). Based on clonal relatedness of prevalent LGV strains, there is evidence that the LGV epidemic among MSM prevailed already in the United States in the 1980s and was introduced into Europe by the end of the last century via the highly internationalised network of sexual contacts among MSM. A new LGV variant was unveiled and designated L2c.

\section{S16.5 MANAGEMENT OF SYPHILIS IN PREGNANCY}

doi:10.1136/sextrans-2013-051184.0077

J Wilson. Centre for Sexual Health, Leeds, UK

There are a number of clinical challenges that are specific to managing syphilis in pregnancy: Which women have the highest risk of adverse pregnancy outcome and is there anything extra we should do for them? What effect does the timing of treatment have on the pregnancy? What is the best treatment and should this be modified in the presence of HIV? What effect does the Jarisch-Herxheimer reaction have on pregnancy? What rate of adverse pregnancy outcome can be expected following successful treatment? Should all babies be treated at birth and how should the baby be monitored?

Early stage maternal infection and higher RPR increase the risk of adverse pregnancy outcome. Treatment in the third trimester is also associated with poorer outcomes. Parenteral penicillin G is the only recommended therapy for treatment of syphilis during pregnancy, and the lack of effective alternatives is why desensitisation is recommended in those who report a penicillin allergy. However, a metaanalysis concluded there is insufficient evidence to determine an optimal penicillin regimen. Adequate treatment in pregnancy significantly reduces adverse pregnancy outcomes (APOs) and congenital syphilis but APOs are still reported probably due to placental damage and effects of the fetal immune response. Some guidelines recommend treating all infants born to positive women whether or not the mother was adequately treated in pregnancy whereas others suggest this is probably not necessary. All recommend examination and serological testing of the babies every 3 months until the test/s become nonreactive.

This presentation will look at the evidence base, and the recommendations in different national guidelines, to try to provide answers to these questions.

\section{YI - American Sexually Transmitted Diseases Association - Young Investigators Symposium: research in progress: Highlights from the American STD association developmental awards programme}

\section{YI.1 CONDOM USE \& PLEASURE IN A SAMPLE OF YMSM: A CONCEPTUAL FRAMEWORK}

doi:10.1136/sextrans-2013-051184.0078

${ }^{1}$ R Arrington-Sanders, ${ }^{2} \mathrm{G}$ W Harper, ${ }^{3} \mathrm{~J} D$ Fortenberry, ${ }^{2} \mathrm{~J}$ A Bauermeister. ${ }^{1}$ Division of General Pediatrics \& Adolescent Medicine, Johns Hopkins School of Medicine, Baltimore, MD, United States, ${ }^{2}$ Health Behavior and Health Education, University of Michigan School of Public Health, Ann Arbor, MI, United States, IIndiana University School of Medicine Section of Adolescent Medicine, Indianapolis, IN, United States 